\title{
Non-linear temperature response of Bragg gratings in doped and un- doped holey polymer optical fibre
}

\author{
K. Kalli ${ }^{\mathrm{b}}$, D.J. Webb ${ }^{\mathrm{a}}$, K. Carroll ${ }^{\mathrm{a}}$, C. Zhang ${ }^{\mathrm{a}}$, Alex Argyros ${ }^{\mathrm{c}}$, Maryanne Large ${ }^{\mathrm{c}}$, Martijn van \\ Eijkelenborg ${ }^{\mathrm{c}}$

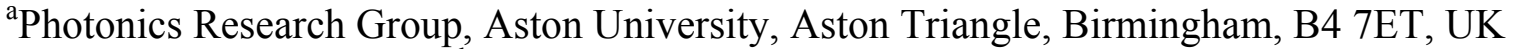 \\ ${ }^{b}$ Higher Technical Institute, Cyprus \\ ${ }^{c}$ Optical Fibre Technology Centre, University of Sydney, Australia
}

\begin{abstract}
We present measurements on the non-linear temperature response of fibre Bragg gratings recorded in pure and trans-4stilbenemethanol-doped polymethyl methacrylate (PMMA) holey fibres.
\end{abstract}

Keywords: Polymer optical fibre, microstructured optical fibre, fibre Bragg gratings, temperature effects, non-linearity

\section{INTRODUCTION}

At the moment there is considerable interest in the use of polymer optical fibre (POF) in a variety of applications. For example, in several countries POF is seen as a good candidate as a digital transmission medium for local area networks or fibre to the home. ${ }^{1}$ Bragg gratings are increasingly used as sensors in optical fibre networks for a number of applications particularly for temperature, pressure and strain measurements. ${ }^{2}$ Temperature measurements are of special interest, either for direct temperature sensing or for multi-measurand sensing where the primary measurement field may not be temperature, but where the measurement is temperature compensated, for example in quasi-static strain measurement systems. In both cases accurate temperature measurements depend on knowing the wavelength-totemperature response of the grating. There is growing interest in the potential applications of fibre Bragg gratings (FBGs) recorded in polymer optical fibre (POF) in order to both extend the temperature measurement range and take advantage of the increase in thermal sensitivity ${ }^{34}$. Additionally, the ability to survive very high strains coupled with the inherent material biocompatibility and the intrinsic flexibility provided by organic chemistry are key advantages over silica fibre.

\section{MPOF GRATING FABRICATION}

The fibre used in this work is fabricated in the Optical Fibre Technology Centre at the University of Sydney, Australia. It was made from a PMMA primary preform of $70 \mathrm{~mm}$ diameter, into which the desired pattern of holes was drilled. The primary preform was drawn to form the $10 \mathrm{~mm}$ diameter secondary preform, and the secondary preform was drawn to fibre directly, without sleeving. The endlessly single mode microstructured polymer optical fibre (mPOF) with flat sides has an outside diameter of $150 \mu \mathrm{m}$ (round edges) and $115 \mu \mathrm{m}$ (flat edges) and a core diameter of $20 \mu \mathrm{m}$. The core being surrounded by 60 air holes with diameters of $2.6 \mu \mathrm{m}$ and a separation of $8.6 \mu \mathrm{m}$, see Figure 1 . The ends of the mPOF were cleaved by using a hot sharp razor blade ${ }^{5}$. The mPOF was then mounted in v-grooves which were attached to an $\mathrm{x}, \mathrm{y}, \mathrm{z}$ translation stage, allowing the adjustment of coupling between fibre and connector. Figure 2 shows the inscription set-up based on the standard phase mask technique. A continuous wave $325 \mathrm{~nm}$ Kimmon helium cadmium laser was used to inscribe the FBG. A $10 \mathrm{~cm}$ focal length plano-convex cylindrical lens was mounted in the system to focus the light down to the core of $\mathrm{mPOF}$. The Phase mask (O/E land) used in experiment was of $1057.2 \mathrm{~nm}$ pitch, which produced an FBG around $1562 \mathrm{~nm}$ Bragg wavelength.

Photonic Crystal Fibers, edited by Kyriacos Kalli, Proc. of SPIE Vol. 6588,

65880E, (2007) - 0277-786X/07/\$18 · doi: 10.1117/12.722634 


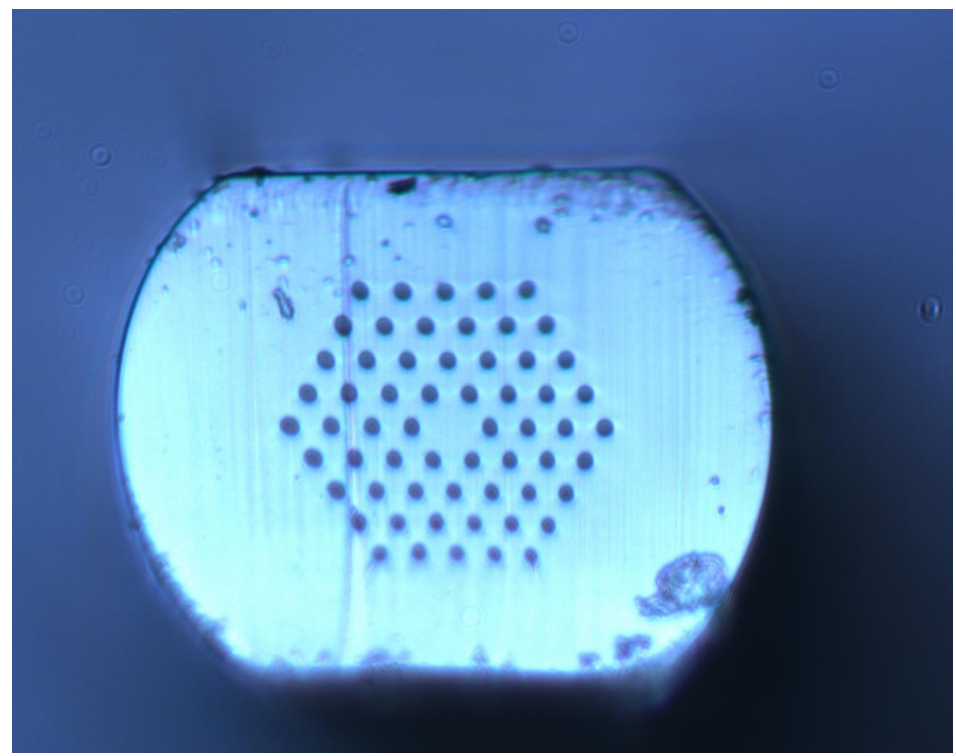

Figure 1: Optical microscope image of the cross-section of the mPOF.

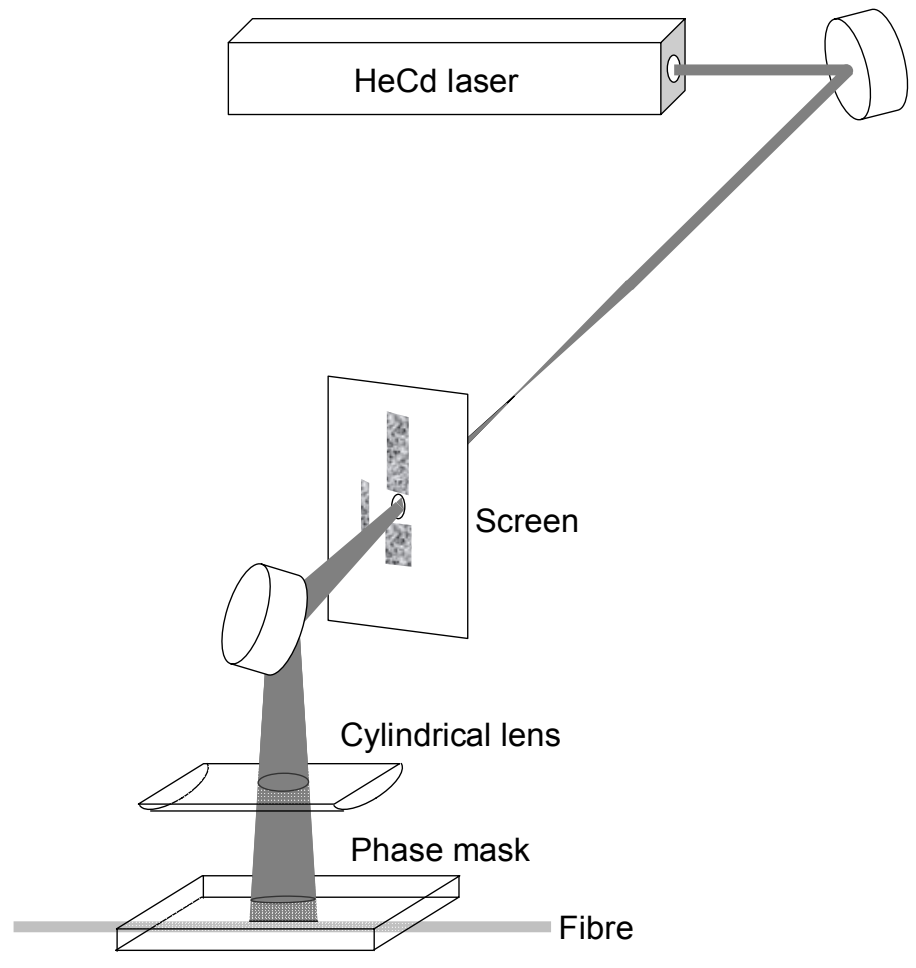

Figure 2 Experiment Setup

In the experiment we found the alignment of $\mathrm{mPOF}$ can considerably affect the quality of the grating, which is quite different from FBG fabrication in silica optical fibre. Figure 3 shows the back-refection pattern of the laser beam from the mPOF. To get the strongest grating, the pattern must be made very thin and bright (the left part of the figure) by adjusting the $\mathrm{mPOF}$; this procedure probably involves eliminating any bends in the fibre. 

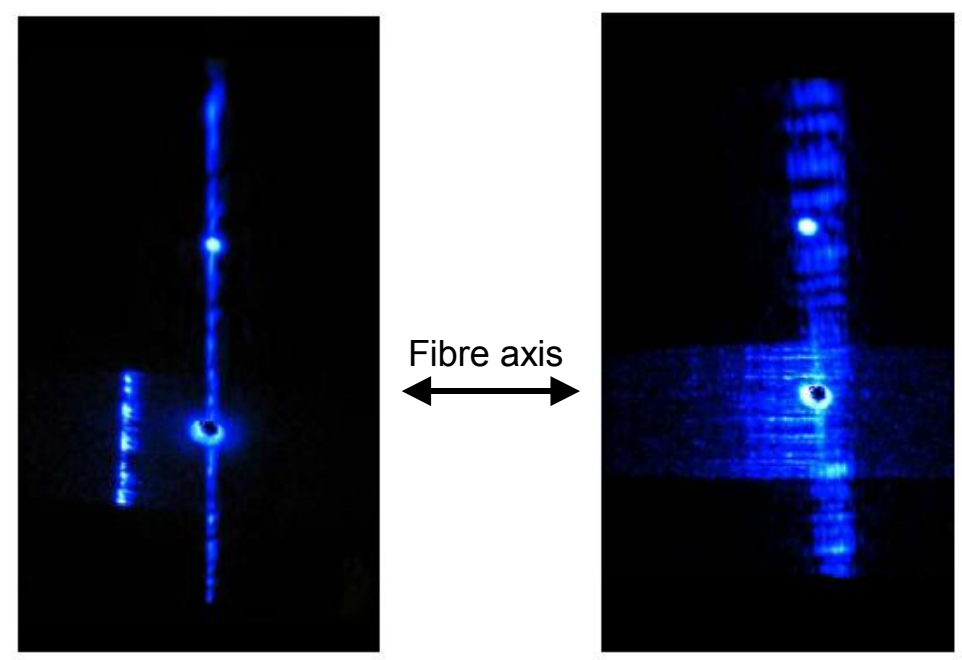

Figure 3: Backscattered diffraction patterns (the left pattern was needed for successful inscription). ${ }^{6}$

After the alignment of the mPOF, light was coupled into the fibre to monitor the grating using the setup shown in Figure 4. A $1550 \mathrm{~nm}$ SMF coupler was used to monitor the grating in reflection. One output arm of the coupler was spliced with an FC/APC pigtail which was used to couple the light into the mPOF. Between the AFC connector and the mPOF, a small drop of index matching gel was used to reduce the Fresnel reflections and help with coupling the light from connector to fibre. The two input arms of the coupler, spliced with FC/PC pigtails were correspondingly connected with the light source and optical spectrum analyser (OSA). At first, the light source was a He-Ne laser working at $633 \mathrm{~nm}$. By adjusting the translation stage, the maximum throughput of $\mathrm{mPOF}$ at $633 \mathrm{~nm}$ was obtained. Then the light source was switched to a 1530-1610nm range ASE light source (Thorlabs ASE-FL7002-C4), matched to the expected Bragg wavelength of the grating. Finally, the phase mask was placed on two pieces of polyimide tape which are used to hold the fibre on the stage. In our experiment, we found that three layers of tape create the roughly right distance between the fibre and phase mask (around $80 \mu \mathrm{m}$ ).

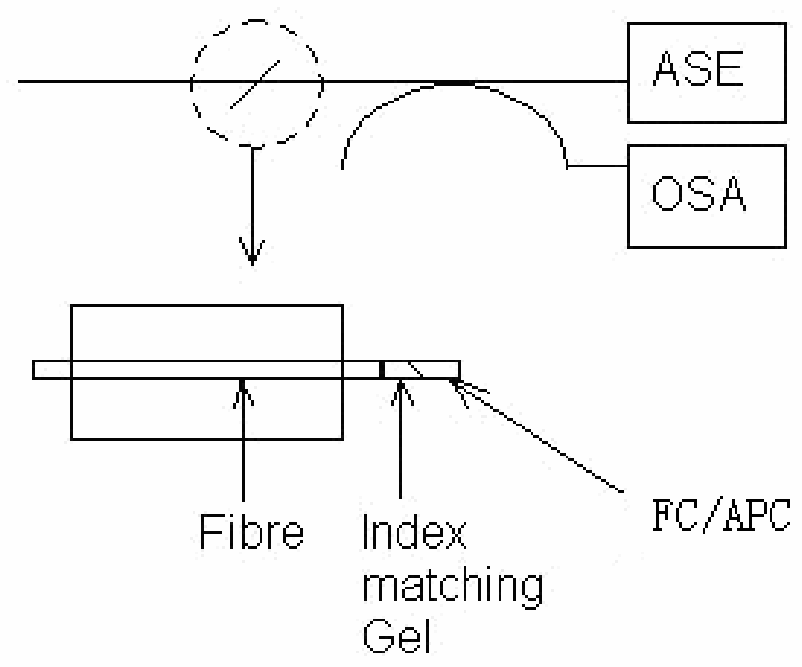

Figure 4: Diagram of grating monitoring arrangement. 
Normally it required around 30-40 minutes of exposure to approach a maximum grating reflectivity. The final spectra from two FBGs are shown in figure 5.

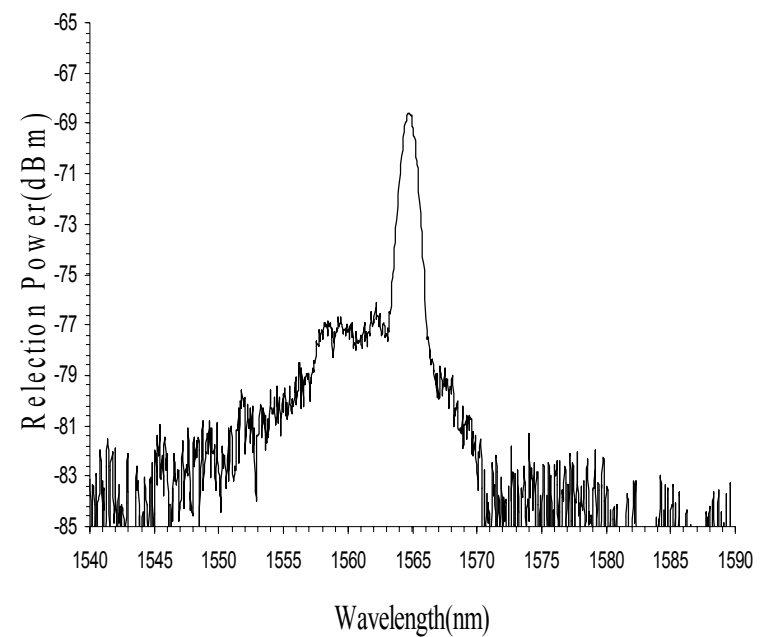

Grating 1

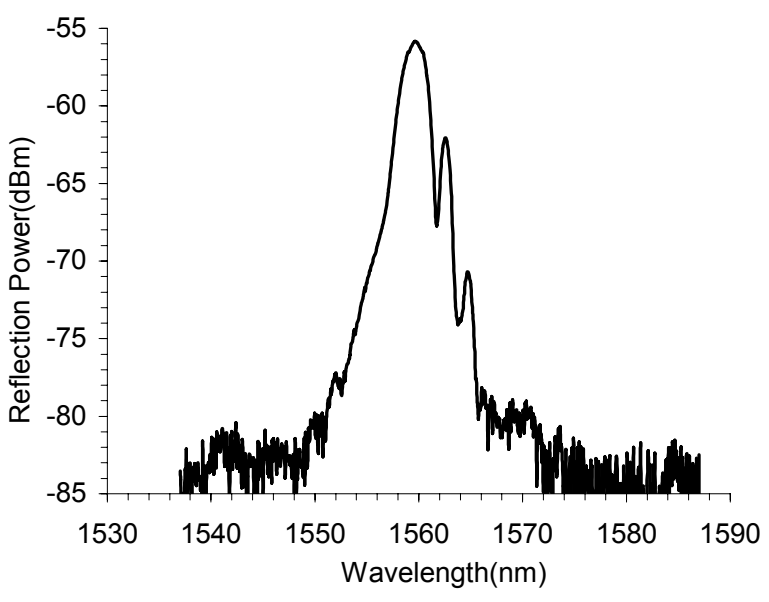

Grating 2

Figure 5 Reflection spectrum of mPOF grating

\section{NONLINER THERMAL RESPONSE OF MPOF GRATINGS}

\subsection{Thermal Response of undoped mPOF Gratings}

To investigate the thermal response of the undoped mPOF grating, an arrangement shown in Figure 6 was used. Two vgrooves were fastened on the translation stage leaving a $1 \mathrm{~cm}$ long air gap between them. The mPOF with an inscribed FBG (Grating 1 in Figure 5) was placed in the v-grooves making sure the FBG was located in the air gap, thus preventing heat exchange between FBG and metal v-grooves. A high power resistor $(25 \mathrm{~W}, 100 \Omega$,) was placed over the FBG to heat the device. A thermocouple was stuck on the resistor to monitor the temperature change. In addition, the whole heating zone was covered by polystyrene pieces to provide an equilibrium temperature surrounding the grating.

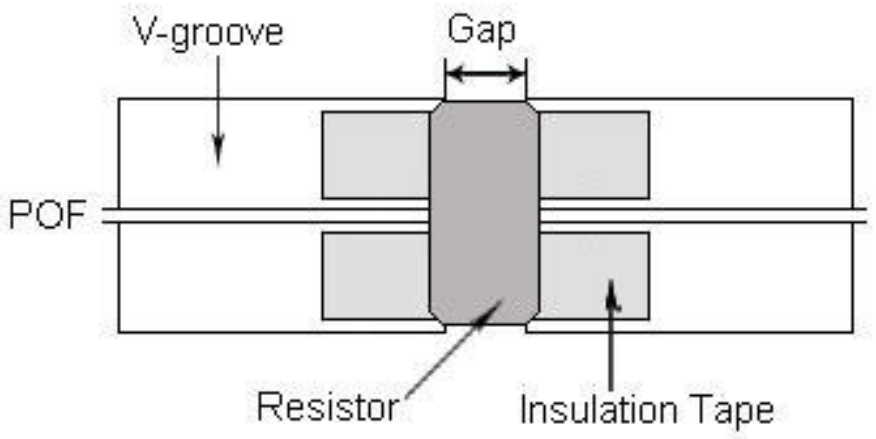

Figure 6: mPOF FBG temperature calibration setup

By changing the voltage value across the resistor, the region containing the FBG was then heated to several temperatures, with the voltage held constant for 10 minutes to ensure thermal equilibrium. During this period, we recorded the value of the peak shift of the grating spectrum and at the end of the set of readings the resistor was allowed to cool down to room temperature. This process was repeated 3 times, each time with the heating ending at a higher temperature. The results are shown in Figure 7. 


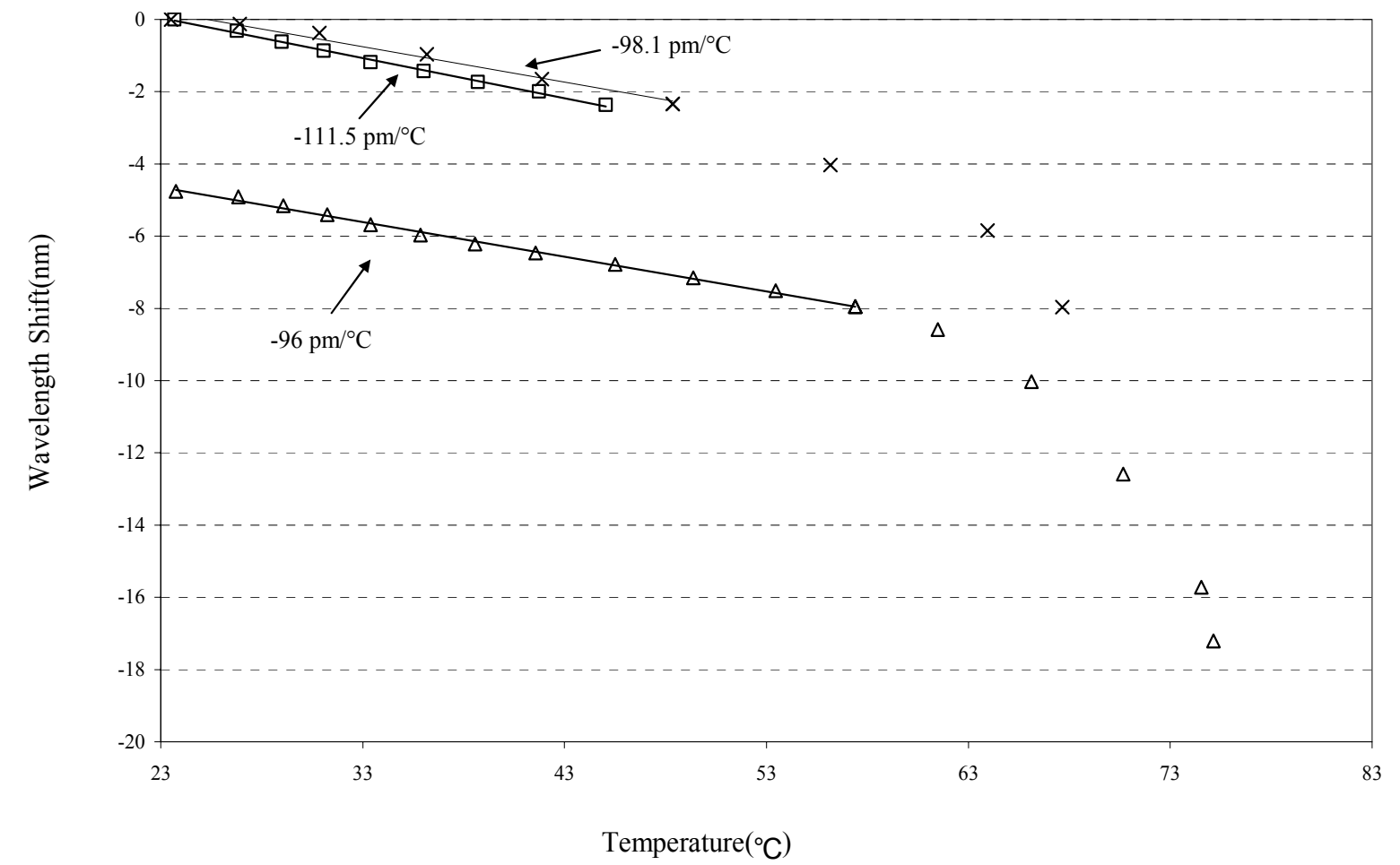

Figure 7: Bragg wavelength shift with temperature of grating 1 for three consecutive heating cycles. $\square$ First cycle, $x$ second cycle, $\Delta$ third cycle.

From the results, we can observe a non-linear negative wavelength shift leading to significant deviation from linearity for temperatures above about $50^{\circ} \mathrm{C}$. These results differ significantly from those for step index polymer optical fibre reported by Liu et $a l^{3}$ and also differ from the linear temperature response of silica optical fibre. In those two kinds of fibre, the Bragg wavelength shift $\Delta \lambda_{\bar{\theta}}$ of a grating as a function of temperature change $\Delta \mathrm{T}$ can be expressed as a linear equation ${ }^{7}$ :

$$
\Delta \lambda_{\bar{s}}=\lambda_{\bar{s}}(\alpha+\beta) \Delta \mathrm{T}
$$

where $\alpha$ is the thermal expansion coefficient, $\beta$ is the thermo-optic coefficient. However this equation cannot be used to explain the behaviour here. Note that even in the region below $50^{\circ} \mathrm{C}$, which we refer to as quasi-linear, there is evidence that the response is more properly modelled by a quadratic.

We found the Bragg wavelength shift with temperature curve of heating cycles 2 and 3 can be separated into two parts, the quasi-linear region followed by a steep drop in Bragg wavelength. The temperature at which the responsivity increases markedly depends on the previous thermal history of the fibre. For a new grating the temperature is around $50^{\circ} \mathrm{C}$, but if the grating has been previously heated beyond that temperature then the quasi-linear region extends up to the previous highest temperature experienced by the grating This annealing process is accompanied by a permanent room temperature shift in the Bragg wavelength that can be as much as several $\mathrm{nm}$. The temperature sensitivity of the grating in the quasi-linear region seems to reduce slightly after annealing to progressively higher temperatures. There appears to be a link between the hysteresis in the Bragg wavelength and a permanent reduction in the fibre length that we have observed after heating the fibre. The fibre drawing process introduces some alignment of the polymer molecules, to a degree which depends on the drawing tension ${ }^{8}$. Heating the the fibre to a certain point permits a relaxation back to a more disordered structure accompanied by a reduction in length and an increase in diameter. 
To explore the repeatability of these nonlinear responses, another newly fabricated grating was used (Grating 2 in figure 5 ) and the heating temperature range was extended to near the glass transition temperature. The results are shown in Figure 8. The results are similar to the previous experiment except that with the higher temperature the effects of hysteresis are more clearly visible. The permanent negative shift in the room temperature Bragg wavelength was almost $20 \mathrm{~nm}$ after heating to around $85^{\circ} \mathrm{C}$.

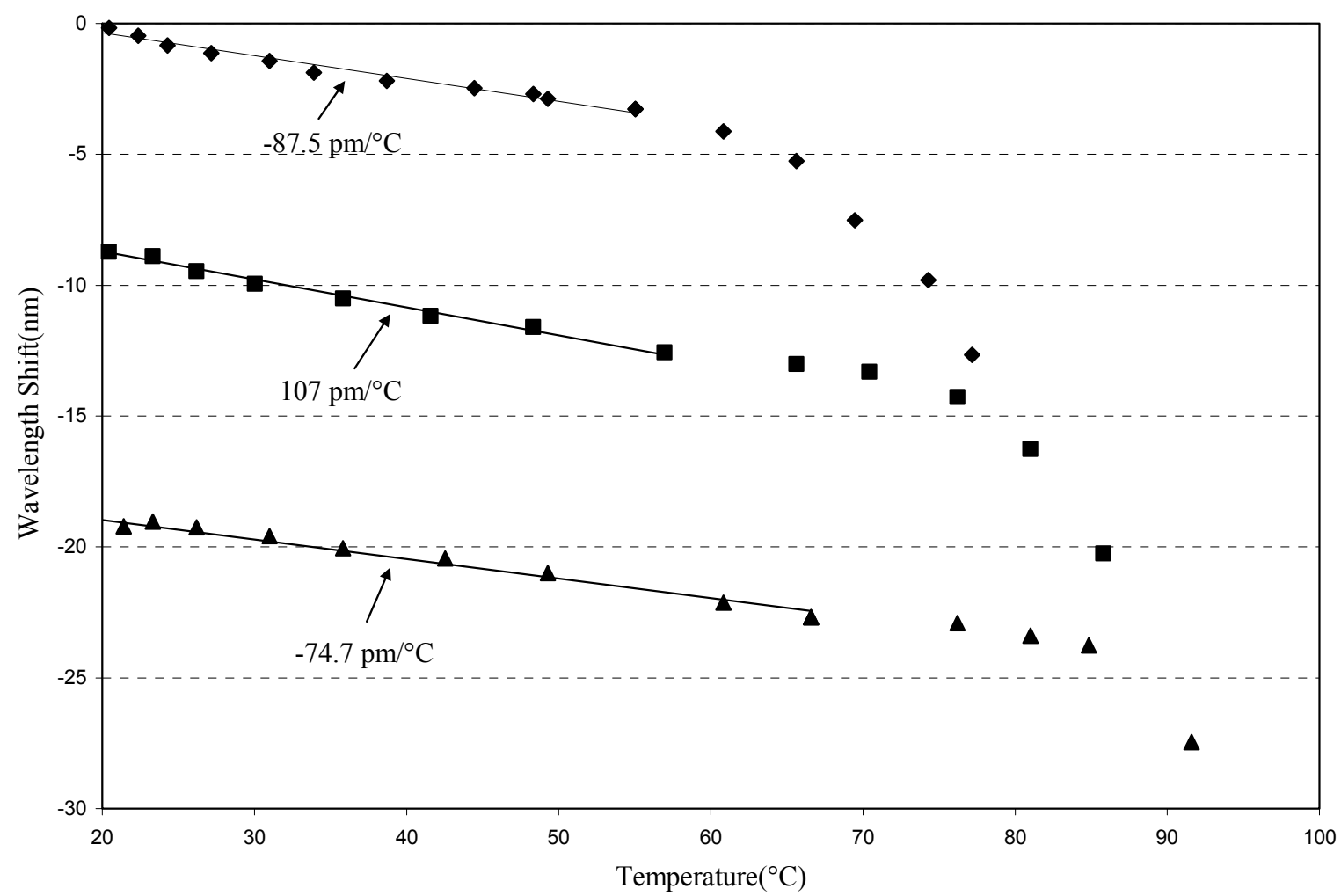

Figure 8: Bragg wavelength shift with temperature of grating 2 for three consecutive heating cycles. • First cycle, a second cycle, $\Delta$ third cycle.

\subsection{Thermal Response of doped mPOF Gratings}

Trans-4-stilbenemethanol is a stilbene derivative compound that exhibits a reversible trans- to cis-photoisomerization. ${ }^{9}$ Due to trans- to cis-isomerization, PMMA with this dopant show a significant photo-induced change of refractive index upon $325 \mathrm{~nm}$ UV irradiation. ${ }^{10}$ As the isomerisation process can be initiated by weak UV irradiation, comparatively lower photon energy and shorter exposure times during grating inscription are expected than for pure PMMA fibre. ${ }^{11} \mathrm{~A}$ grating was inscribed in a single mode mPOF doped with trans-4-stilbenemethanol. Figure 9 shows the grating profile. To investigate the thermal response of the doped $\mathrm{mPOF}$ grating, the same experimental configuration described in 3.1 was used. On heating the grating we observed a non-linear negative wavelength shift, similar to the results for undoped mPOF gratings. From the result shown in Figure 10, we found that for this fibre the turning point appeared at around $60^{\circ} \mathrm{C}$. 


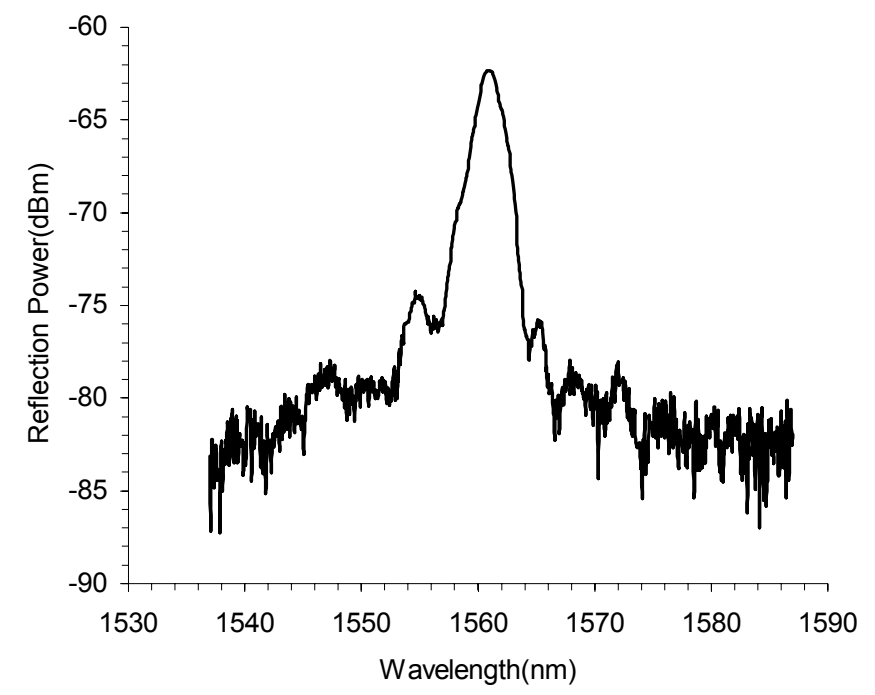

Figure 9: Spectrum of FBG in trans-4-stilbenemethanol doped mPOF.

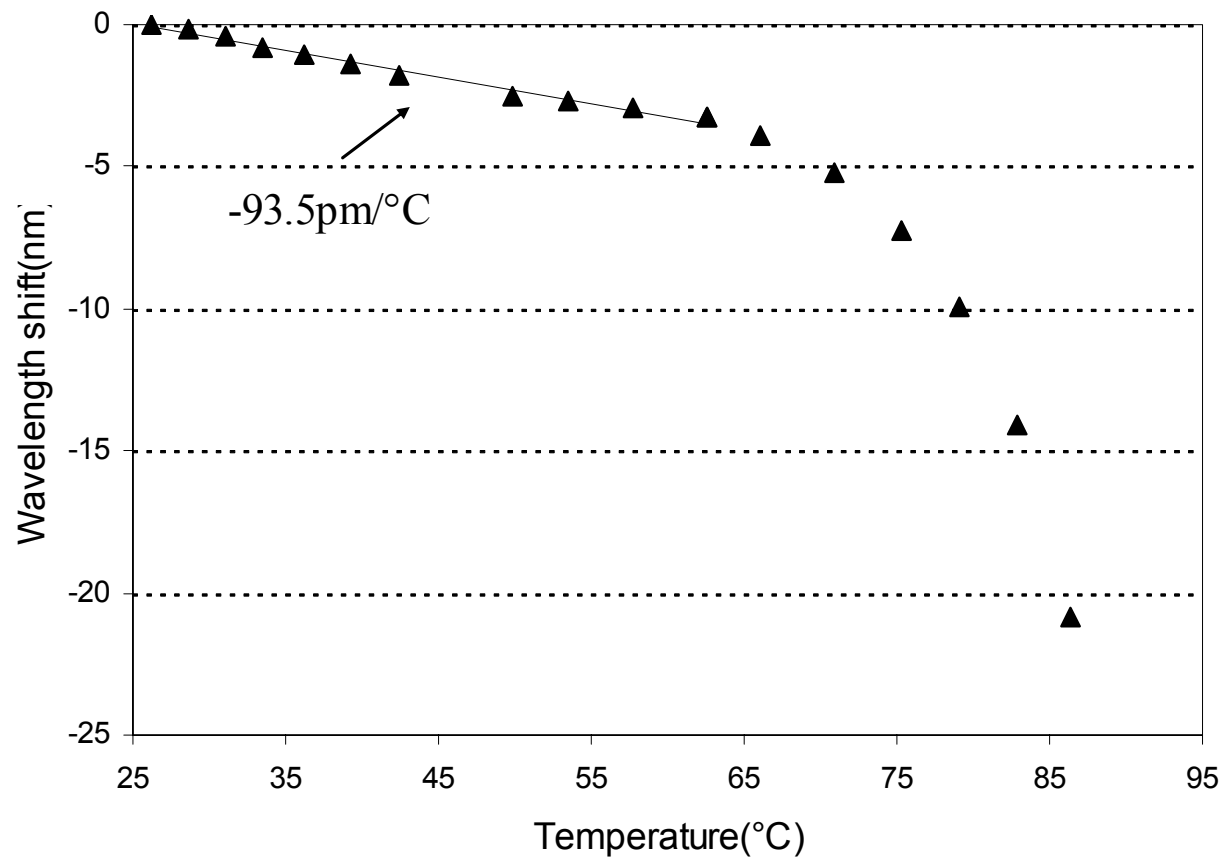

Figure 10: Bragg wavelength shift with temperature of grating in trans-4-stilbenemethanol doped mPOF.

\section{CONCLUSIONS}

We have investigated the thermal sensitivity of the Bragg wavelength of FBGs recorded in pure and doped mPOF. A newly produced grating only has a limited linear, reproducible temperature range, up to around $50^{\circ} \mathrm{C}$ in the case of pure PMMA. We have discovered that this range may be usefully extended to in excess of $80^{\circ} \mathrm{C}$ by annealing the fibre up to 
that temperature before use. This annealing process is accompanied by a permanent negative wavelength shift of almost $20 \mathrm{~nm}$, which must be taken into account when the phase mask period is being chosen.

\section{REFERENCES}

${ }^{1}$ W. Daum, J. Krauser, P. E. Zamzow, and O. Ziemann POF Polymer Optical Fibers for Data Communication (Springer-Verlag, Berlin, 2002)

${ }^{2}$ A. D. Kersey, M. A. Davis, H. J. Patrick, M. LeBlanc, K. P. Koo, C.G. Askins, M. A. Putnam, and E. J. Friebele, "Fiber grating sensor,” J. Lightwave Technology, 15, 1442-1463, 1997

${ }^{3}$ H. Y. Liu, G. D. Peng and P. L. Chu, "Thermal tunability of polymer optical fibre Bragg gratings", IEEE Photonic Technology Letters, Vol.13, No.8, pp.824-826, 2001

${ }^{4}$ K. Kalli, H. L. Dobb, D. J. Webb, K. Carroll, M. Komodromos, C. Themistos, G. D. Peng, Q. Fang, and I. W. Boyd, "Electrically tunable Bragg gratings in single-mode polymer optical fibre", Optics Letters, 32, 3, 214-216, 2007

${ }^{5}$ S.H. Law, J.D. Harvey, R.J. Kruhlak, M. Song, E. Wu, G.W. Barton, M.A. van Eijkelenborg, M.C.J. Large, "Cleaving of microstructured polymer optical fibres", Optics Communications, 258, 2, 193-202, 2006

${ }^{6}$ H. Dobb, D. J. Webb, K. Kalli, A. Argyros, M. C. J. Large and M. A. van Eijkelenborg, "Continuous wave ultraviolet light-induced fiber Bragg gratings in few- and single-mode microstructured polymer optical fibers", Optic Letters, 30, 24, 3296-3298, 2005

${ }^{7}$ A. Othonos and K. Kalli Fiber Bragg gratings: fundamentals and applications in telecommunications and sensing (Artech House, 1999)

8 Takaaki Ishigure, Miki Hirai, Masataka Sato, Yasuhiro Koike, “Graded-Index Plastic Optical Fiber with High Mechanical Properties Enabling Easy Network Installations. I," Journal of Applied Polymer Science, Vol. 91, 404-409, 2004

${ }^{9}$ G. H. Brown Photochromism (Wiley, New York, 1971)

${ }^{10}$ J. Yu, X. Tao, H. Tam, D. Yang, M. Suleyman Demokan, " Photosensitivity and grating development in trans-4-stilbenemethanoldoped poly(methyl methacrylate) materials", Optics Communications, 265, 1, 132-139, 2006

11 J.M.Yu, X.M. Tao, H.Y. Tam, "Trans-4-stilbenemethanol-doped photosensitive polymer fibers and gratings", Optic Letters, 29, 2 , 2004 